\title{
Lymphovenous Bypass
}

National Cancer Institute

\section{Source}

National Cancer Institute. Lymphovenous Bypass. NCI Thesaurus. Code C159300.

A surgical procedure in which anastomoses are created from lymphatic vessels to veins.. 